\title{
Beneficial impact of a thin tunnel barrier in quantum well intermediate-band solar cell
}

\author{
Nicolas Cavassilas ${ }^{1,2, *}$, Daniel Suchet ${ }^{3,2}$, Amaury Delamarre $^{3,2}$, Fabienne Michelini $^{1}$, Marc Bescond $^{4}$, \\ Yoshitaka Okada $^{3,2}$, Masakazu Sugiyama ${ }^{3,2}$, and Jean-Francois Guillemoles ${ }^{2,5}$ \\ 1 Aix Marseille Université, CNRS, Université de Toulon, IM2NP UMR 7334, 13397 Marseille, France \\ 2 NextPV, LIA, CNRS-RCAST/U, Tokyo-U, Bordeaux, Tokyo 153-8904, Japan \\ ${ }^{3}$ Research Center for Advanced Science and Technology, The University of Tokyo, Tokyo 153-8904, Japan \\ ${ }^{4}$ LIMMS, CNRS-Institute of Industrial Science, UMI 2820, University of Tokyo, Tokyo 153-8505, Japan \\ ${ }^{5}$ IRDEP, UMR 7174 CNRS EDF Chimie ParisTech, EDF R\&D, Chatou, France
}

Received: 25 July 2018 / Received in final form: 16 October 2018 / Accepted: 19 October 2018

\begin{abstract}
Based on electronic quantum transport modeling, we study the transition between the intermediateband and the conduction-band in nano-structured intermediate-band solar cell. We show that a tunnel barrier between the quantum well (QW) and the host material could improve the current. The confinement generated by such a barrier favors the inter-subband optical coupling in the QW and then changes the excitation-collection trade-off. More surprisingly, we also show that tunneling impacts the radiative recombination and then the voltage. Using a detailed balance model we explain and we propose a broadening factor for this $V_{\text {oc }}$ modification. Finally we show that a thin tunnel barrier is beneficial for both current and voltage.
\end{abstract}

Keywords: intermediate band solar cell / quantum modeling / quantum structures / intraband transition

\section{Introduction}

By introducing an intermediate band (IB) in a wide bandgap solar cell, the aim is to increase the short circuit current $I_{\mathrm{sc}}$ without reducing the open-circuit voltage $V_{\mathrm{oc}}$ $[1,2]$. The current increases as the IB acts as a built in up-converter to generate an electron hole pair with two low-energy photons. To limit detrimental effect voltage drop, the IB must be electrically isolated from the contacts of the cell so that the recombination is still controlled by the wide bandgap. This theoretically allows to exceed the Shockley-Queisser (SQ) efficiency limit [3] with a $V_{\mathrm{oc}}$ controlled by the wide bandgap while low-energy photons generate electron-hole pairs.

Yet practically, this concept has not yet resulted in a cell exceeding the SQ limit $[4,5]$. However, IB solar cell (IBSC), generally based on quantum structures, still offers characteristics that are often lower than that of the same cells without IB [6]. To address this issue the increase of current [7] is not enough to catch up with the degradation of the $V_{\text {oc }}$. It is difficult to obtain a splitting between the Fermi levels of the IB and the contact (usually the n-type contact) because the electrons easily relax from the

\footnotetext{
* e-mail: nicolas.cavassilas@im2np.fr
}

conduction band of the wide bandgap material to the IB. In other words, the IB is not well isolated from contacts and $V_{\mathrm{oc}}$ is controlled by recombination across a narrow bandgap.

Furthermore, the increase of current remains low as it is difficult to have simultaneous efficient photon absorption and a fast collection of the excited electrons. Indeed, if the IBSC is based on quantum structures, the electrons in IB are confined in localized states. The optical transition between the IB and the conduction band is then efficient if the excited state is also localized. This improves the wavefunction overlap and the corresponding intraband (intersubband) transition [8,9]. However, if the excited electron is strongly confined in the quantum structure, it cannot reach the conduction band to be collected. It will finally relax by emitting photon and/or phonons. As a result the choice of the coupling between the excited state of the quantum structures and the conduction band of the wide bandgap is crucial to have a good excitation-collection trade-off. In case of bound-to-bound absorption with a thick tunnel barrier the absorption is high, but the electrons collection is limited. In a bound-to-continuum absorption without tunnel barrier the collection is fast but the absorption is low. In this letter we propose a comprehensive analysis of this problem. We will see that a thin tunneling barrier between the quantum structure 
and the wide bandgap material offers a good excitationcollection trade-off. This permits to optimize the current generated by the intraband transition of an IBSC. More unexpected, we will show that such a coupling also modifies the photon emission in the intraband transition and then impacts the $V_{\mathrm{oc}}$.

\section{Model and system}

This theoretical study is based on a quantum electronic transport model in the non-equilibrium Green functions (NEGF) framework. This model allows to consider the quantum effects, such as the confinement, the tunneling, the broadening due to the coupling and the scattering with the photons and the phonons. Nevertheless, except when this will be explicitly stated, we will not consider scattering with phonons. Such a radiative limit permits to facilitate the understanding of the results. The model used is described in reference [10] but as suggested in reference [11] we now consider the non-local character of the electronphoton interaction, which guarantees compliance with the selection rules.

The modeled system is schematically shown Figure 1. We consider a quantum well (QW) between two contacts. The left contact can only inject and collect electrons over an interval of $0.2 \mathrm{eV}$ around the left Fermi level $\mu_{L}$. This value is a bit larger than the broadening of the ground state of the QW, which mimics the IB. Although this is another important issue in IBSC, the optical transition between the valence band and the IB is not treated in this work. In our model we consider this issue solved by assuming the Fermi level $\mu_{L}$ located at the maximum of the density-of-states (DOS) of the ground state (middle of the IB). Electrons on this ground state can then interact with photons and reach an excited state. From there, they either relax (and then do not participate in the current) or reach the right contact. This contact, located at an energy $E_{c}$ above $\mu_{L}$, represents the conduction band-edge of the wide bandgap material. By reaching this contact the electrons generate a current that one chooses positive. The right contact can also inject electrons that can reach the QW, relax by emitting a photon and then reach the left contact. This behavior, which corresponds to the relaxation from conduction band to IB, generates a negative current which is all the more important as the right Fermi level $\mu_{R}$ is high. In an IBSC the aim is to have a positive current balance (more excitations than relaxations) while having a higher Fermi level in conduction band $\left(\mu_{R}\right)$ than in IB $\left(\mu_{L}\right)$. Our system, therefore behaves like an intraband solar cell under bias $q V=\mu_{R}-\mu_{L}$ and characterized by a current $I_{\mathrm{sc}}$ and a voltage $V_{\mathrm{oc}}$. Note finally that, with applied bias, we do not modify the band diagram which is always considered in the flat band approximation.

In the following we compare a bound-to-bound system in which a tunnel barrier is assumed between the QW and the right contact to a bound-to-continuum system in which we do not assume any barrier. While for an interband transition the definition of the energy gap seems obvious, this is not so trivial for an intraband-one. Instead, we define a threshold energy $E_{t}$ as the resonant energy of the transition between the IB and the conduction band i.e., the

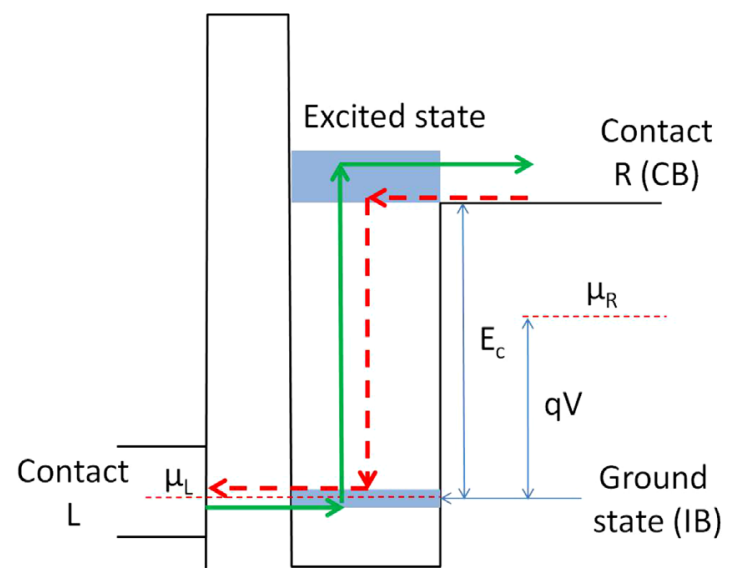

Fig. 1. Schematic representation of the modeled system. The full-arrows show the radiative excitation and the collection while the dash-arrows represent the injection and the emission. We also show the two Fermi levels $\mu_{R}$ and $\mu_{L}$, respectively, corresponding to the IB and the n-type contact. The grey surfaces represent the states.

energy of the maximum absorption considering a blackbody at $6000 \mathrm{~K}$ for the incident photon flux. $E_{t}$ would correspond to the usual energy gap for a system with a step-like absorptivity. For all systems assumed in this work, $E_{t}$ is the same. We chose $E_{t}=1 \mathrm{eV}$, which is largely compared to what is generally considered in IBSC. This choice is based on a study conducted elsewhere [12] which shows that with a ratchet mechanism $[6,13]$ of $0.7 \mathrm{eV}$, such a value is optimal in the case where the absorption is rather narrow as expected in intraband system. The other parameter is the effective mass which equals 0.0665 (in free electron mass). Finally we chose to treat a $\mathrm{QW}$ since our onedimensional model is well adapted to such a system. However, the physical behaviors, such as confinement and absorption are very close in case of quantum dots. Moreover, if it is straigthforward to add a barrier between a QW and a contact, it is also feasible in case of quantum dots assuming a core-shell architecture [14] or dots embedded in specific material [15]. If in the present study we assume a QW, the conclusions can thus be easily extended to the quantum dots.

\section{Results}

\subsection{Current and voltage with the quantum model}

The band diagrams and the local-DOS of the two systems are represented in Figure 2. In both the well thickness is $3.2 \mathrm{~nm}$. In one case, the excited state is a localized state separated from the right contact by a $2 \mathrm{~nm}$-thick tunnel barrier (Fig. 2a). In this system, called bound-to-bound (B-B), $E_{c}$ equals $0.88 \mathrm{eV}$. In the other system the electrons are excited in an energy continuum directly connected to the right contact (Fig. 2b). In the latter case, called boundto-continuum (B-C), in order to have $E_{t}=1 \mathrm{eV}, E_{c}$ is reduced to $0.855 \mathrm{eV}$. In Figure 2, the LDOS clearly shows that in $\mathrm{B}-\mathrm{B}$ the electrons are excited in a localized and narrow quantum state. In $\mathrm{B}-\mathrm{C}$, despite the absence of barrier, we do not obtain a true continuum but rather a strongly broadened state. Indeed, even without barrier, the 


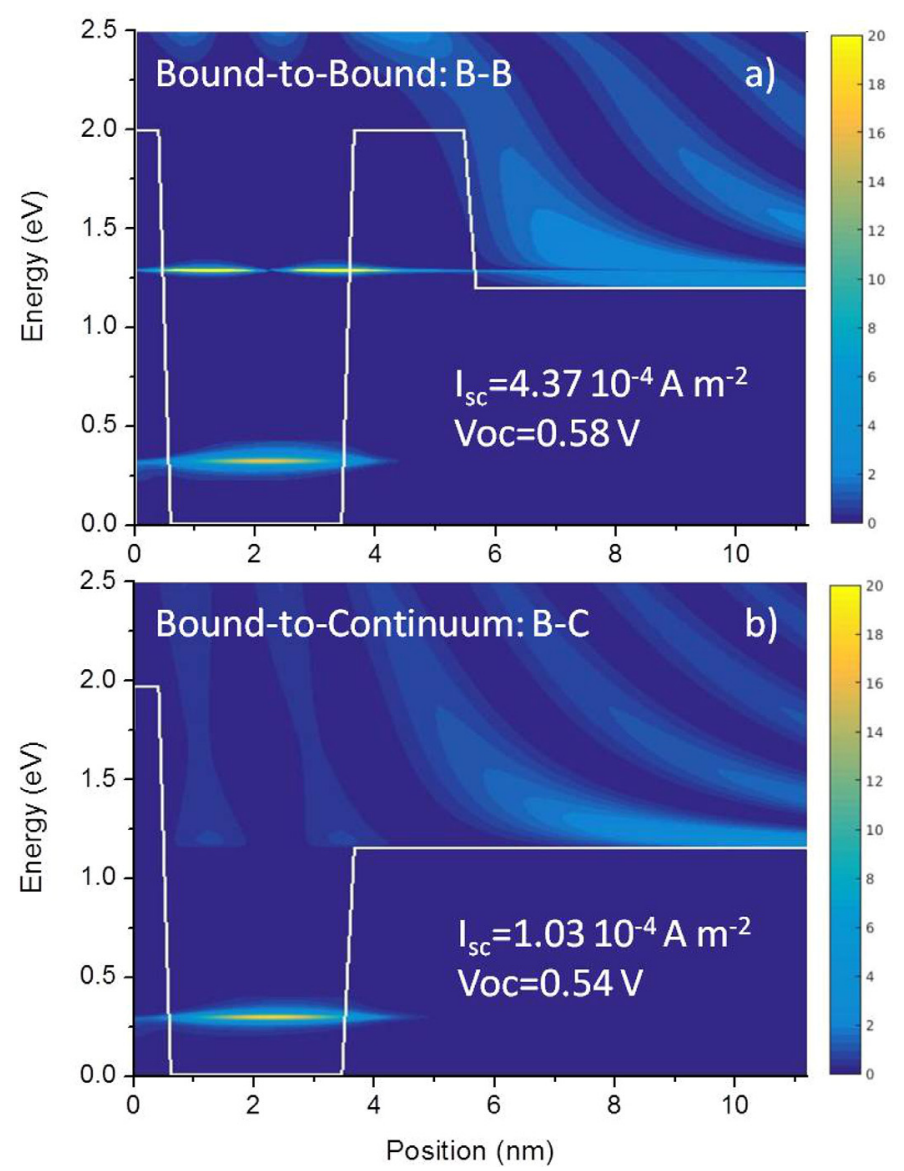

Fig. 2. The band-diagram (white line) and the local-DOS of electrons in (a) the bound-to-bound system and in (b) the bound-tocontinuum system. In a corresponding bound-to-bound IBSC the reduction of voltage due to the presence of the IB should be $1-0.58=0.42 \mathrm{~V}$ against $1-0.54=0.46 \mathrm{~V}$ for the bound-to-continuum configuration.

QW involves quantum reflections and then interferences of the electronic wave-function.

Regarding the results in Figure 2, $I_{\mathrm{sc}}$ and $V_{\mathrm{oc}}$ are both higher in B-B. In order to understand better the results, Figure 3 shows the electronic current versus the photon energy for the two systems. The absorption spectra corresponds to the positive electronic current while the emission is the negative component. The absorption in $\mathrm{B}-\mathrm{B}$ is narrower since, as shown in Figure 2, the excited state is thinner. At the same time the current density is much higher than in B-C's counterpart. A higher absorption in B-B structure, as already shown by the inter-subband absorption coefficient measured in QWs [8] and theoretically observed in quantum dots [16], is not surprising and is due to a larger wave-functions overlap. Finally, the higher generated current in $\mathrm{B}-\mathrm{B}$ is then related to a better tradeoff of the radiative excitation and of the electronic collection. This explains that $I_{\mathrm{sc}}$ is larger in B-B despite the tunneling. However, as it will be confirm in the following, a too thick tunnel barrier degrades the current.

From the emission point of view, we obtain lower threshold energy for B-C. As it will be demonstrated in the following, this behavior, which explains the $V_{\text {oc }}$ degradation in $\mathrm{B}-\mathrm{C}$ is related to the broadening of the excited state. This is particularly detrimental when the excited state is a continuum, which is by definition, strongly broadened.
It is possible to improve $\mathrm{B}-\mathrm{C}$, for example, by reducing the thickness of the well $(2.8 \mathrm{~nm})$. This permits, as shown in Figure 4, to have a quasi-bound state narrower than the strongly broadened state of the B-C. In this new device, called B-Q, by adjusting $E_{c}$ at $0.88 \mathrm{eV}$ in order to have $E_{t}=1 \mathrm{eV}$, the current is largely higher than in the original B-C device, while $V_{\text {oc }}$ is quite similar. Such a current increase is due to a better excitation-collection trade-off. However, the current remains lower than in B-B. Moreover, the fact that in $\mathrm{B}-\mathrm{C}$ and $\mathrm{B}-\mathrm{Q} V_{\mathrm{oc}}$ are close and lower than in B-B, confirms that $V_{\text {oc }}$ is not simply controlled by $E_{c}$. Such results suggest that a narrow excited state is better for $V_{\text {oc}}$. In the following, we propose to use a simpler model in order to verify and explain this counterintuitive feature.

\subsection{Broadening factor in the detailed balance}

We assume a detailed balance model where absorption and emission are calculated assuming a black-body distribution for the photons and a Lorentzian shape for the electronic DOS. Indeed, if we assume an homogeneous broadening due to contact (or others), the DOS spectral shape of states in the QW is given by a Lorentzian distribution [9]. If the tunneling between the excited state and the contact is low (large life-time $\tau$ ) the width $\Gamma$ of the Lorentzian is low following $\Gamma=\hbar / \tau$. We then apply this to the DOS of the 


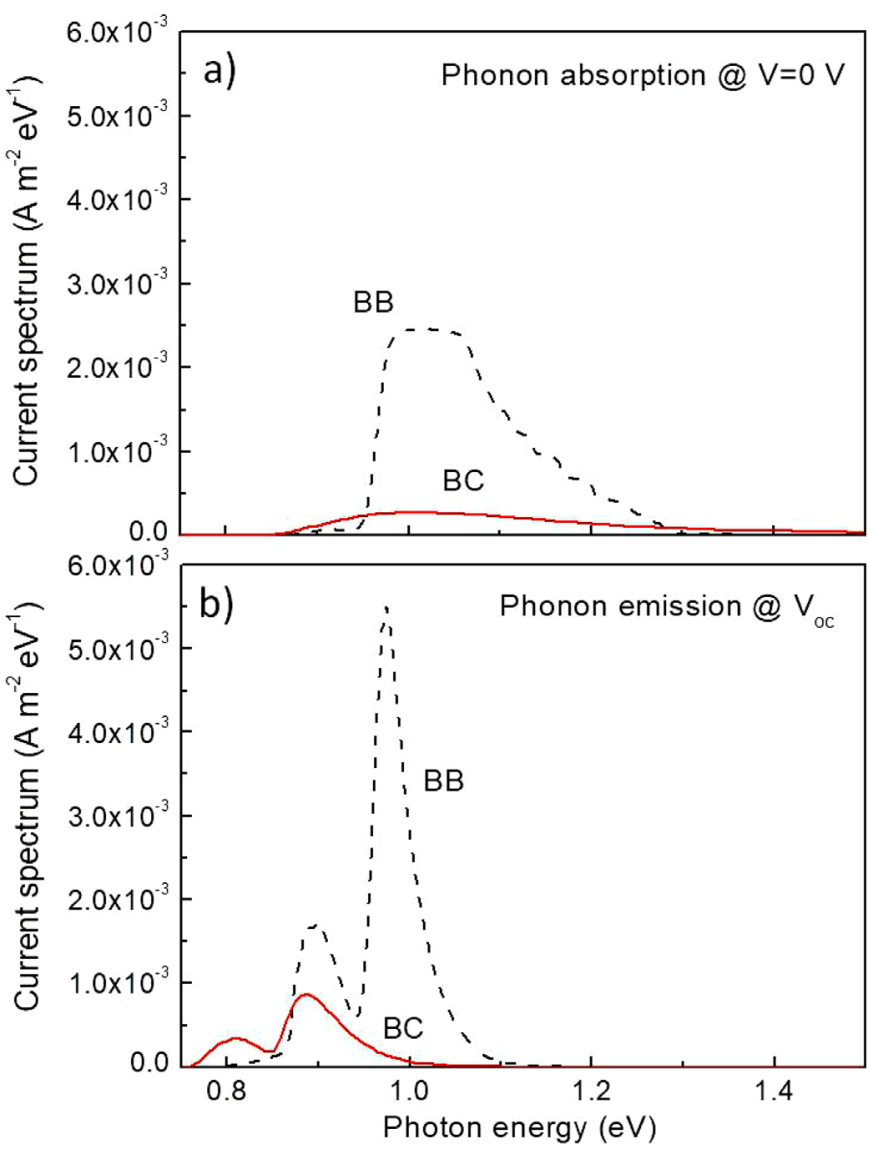

Fig. 3. Current density versus the photon energy generated in each structure B-B and B-C by (a) photon absorption and (b) photon emission.

exited state while, in order to simplify the model, the ground state is assumed as a Dirac function. We finally calculate the current, the emission and the voltage as:

$$
\begin{aligned}
I_{\mathrm{sc}} & =\int_{E_{c}}^{\infty} A_{b}(E) d E \\
& =f M^{2} \int_{E_{c}}^{\infty} \rho\left(E, T_{\mathrm{sun}}, \Delta \mu=0\right) L\left(E, \Gamma, E_{t}\right) d E \\
E_{m} & =\int_{E_{c}}^{\infty} E_{m}(E) d E \\
& =\pi M^{2} \int_{E_{c}}^{\infty} \rho\left(E, T_{\text {cell }}, \Delta \mu=q V\right) L\left(E, \Gamma, E_{t}\right) d E \\
V_{\mathrm{oc}} & =k_{B} T_{\text {cell }} \log \left(\frac{I_{\mathrm{sc}}}{E_{m}(\Delta \mu=0)}\right)
\end{aligned}
$$

with the black-body distribution

$$
\rho(E, T, \Delta \mu)=\frac{2}{h^{3} c^{3}} \frac{E^{2}}{\exp \left(\frac{E-\Delta \mu}{k_{B} T}\right)-1}
$$

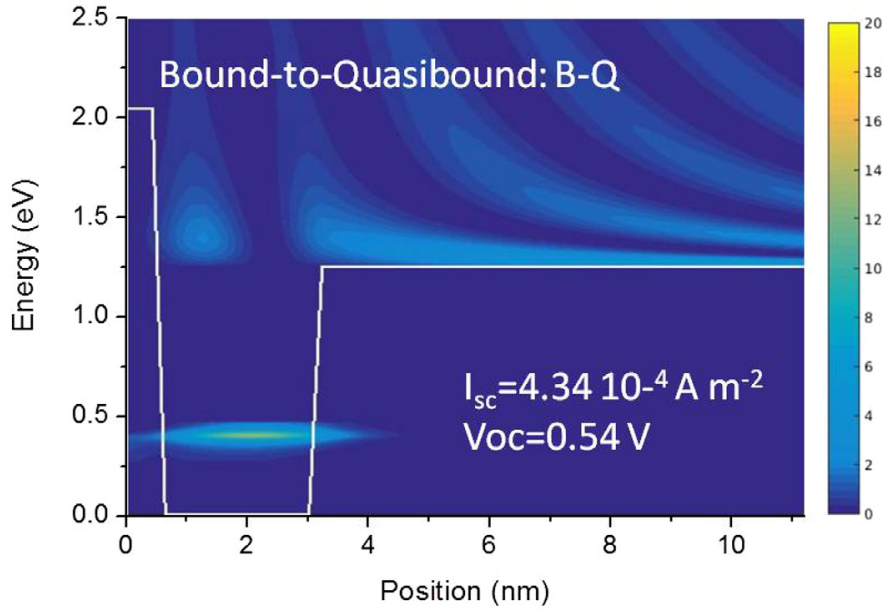

Fig. 4. The band-diagram (white line) and the local DOS of electrons in the bound-to-quasibound system.

and the electronic joined DOS

$$
L\left(E, \Gamma, E_{t}\right)=\frac{\frac{1}{\pi \Gamma}}{1+\left(\frac{E-E_{t}}{\Gamma}\right)^{2}},
$$

where $\Gamma$ is the width, $E_{t}$ the energy of the maximum of the Lorentzian, $q V=\mu_{R}-\mu_{L}$ the bias, $k_{B}$ the Boltzmann constant, and $f=C \times 6.79 \times 10^{-5}$ with $C=1$ the concentration factor. Note that for the integral of $A_{b}$ and $E_{m}$ the minimum of energy is given by $E_{c}$ the band-edge of the right contact. Finally $T_{\text {sun }}$ and $T_{\text {cell }}$ are respectively the temperature of the sun $(6000 \mathrm{~K})$ and of the cell $(300 \mathrm{~K})$. In this model the excitation-collection trade-off is not considered since $M^{2}$, which represents the optical coupling, is taken as a constant and $I_{\mathrm{sc}}$ is directly given by the photon absorption in equation (1). This model is schematically described in Figure 5.

Figure 5 also shows the spectra $A_{b}(E)$ and $E_{m}(E)$ for two values of $\Gamma(5$ and $50 \mathrm{meV})$ with $E_{t}=1 \mathrm{eV}$ and $E_{c}=0.8 \mathrm{eV}$. As obtained with the NEGF model, and although this detailed balance model does not take into account the excitation-collection trade-off, we obtain both $I_{\text {sc }}$ and $V_{\text {oc }}$ larger with a thinner state. As shown in Figure $5, I_{\mathrm{sc}}$ and $V_{\mathrm{oc}}$ are even more degraded than the DOS is high in the QW at the band-edge of the contact $\left[L\left(E_{c}, \Gamma\right.\right.$, $\left.E_{t}\right)$. Indeed, a large $L\left(E_{c}, \Gamma, E_{t}\right)$ means that a large proportion of excited electrons cannot be collected since their energy is lower that the band-edge of the contact. This degrades the current. A large $L\left(E_{c}, \Gamma, E_{t}\right)$ also means a large electronic injection from the contact at low-energy where the emission is very efficient. As already observed in Figure 3, this reduces the energy of the photon emission and then degrades the voltage. Such a behavior, as well as, for example, the reduction of the bandgap by tunneling in ultra thin cells [17], shows that the electronic transport modifies the optical properties of nanoscale devices. Assuming simplified shapes for the functions involved in equations (1) and (2), to analytically solve the corresponding 


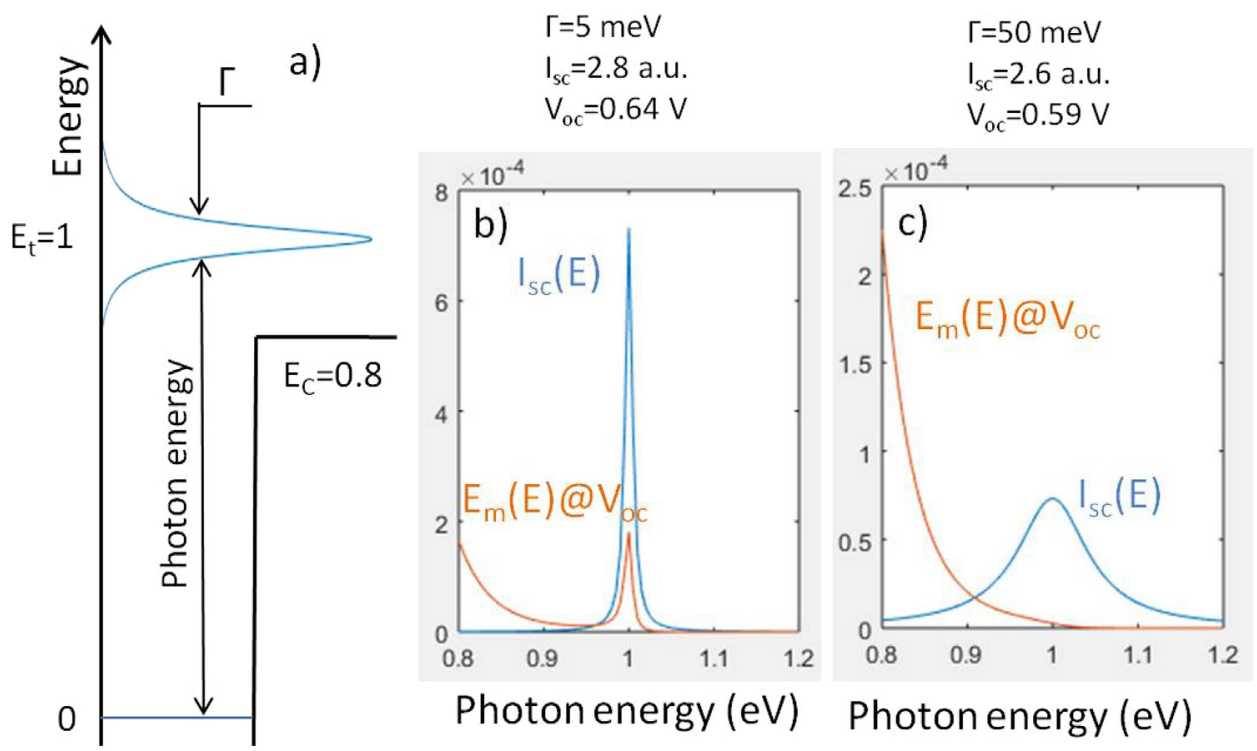

Fig. 5. (a) The schematically described detailed balance model and the corresponding $I_{\mathrm{sc}}$ and $E_{m}$ spectra for (b) $\Gamma=5 \mathrm{meV}$ and for (c) $\Gamma=50 \mathrm{meV}$.

integrals, we obtain this approximate expression for $V_{\mathrm{oc}}$ :

$$
\begin{aligned}
V_{\mathrm{oc}} \simeq & \left(1-\frac{T_{\text {cell }}}{T_{\text {sun }}}\right) E_{t}-\frac{k_{B} T_{\text {cell }}}{q} \log \left(\frac{\pi}{f}\right) \\
& -\frac{k_{B} T_{\text {cell }}}{q} \log (1+A \Gamma),
\end{aligned}
$$

with

$$
\begin{aligned}
A= & \frac{k_{B} T_{\text {cell }}\left(E_{c}^{2}+2 k_{B} T_{\text {cell }} E_{c}+2\left(k_{B} T_{\text {cell }}\right)^{2}\right)}{\pi E_{g}^{2}\left(E_{g}-E_{c}\right)^{2}} \\
& \times \exp \left(\frac{E_{g}-E_{c}}{k_{B} T_{\text {cell }}}\right) .
\end{aligned}
$$

With the parameters assumed to calculate the results shown in Figure 5, the equation (7) gives $A=5274 \mathrm{eV}^{-1}$ to be compared to $5800 \mathrm{eV}^{-1}$ for the value found by fitting the numerical data. The two first terms in the expression of $V_{\mathrm{oc}}$ are the well-known Carnot and Boltzmann factors [18,19] while the third one is relative to an original broadening behavior that we call the broadening factor. The origin of this degradation, as already observed in bulk materials $[20,21]$, is the mismatch between the absorption and the emission energies. This factor is generally not considered in detailed balanced [18] since the absorption is assumed sharp at the bandgap. In intraband system this broadening behavior degrades both $I_{\mathrm{sc}}$ and $V_{\mathrm{oc}}$ when the excited state is too thick like in the bound-to-continuum configuration.

Phonon emission is expected to favor the band-edge photon emission and then to degrade $V_{\text {oc }}$. With our NEGF model we show that such scattering with polar optical phonon is stronger in the continuum than in the QW (and is expected to be even weaker in quantum dot).
The consequence is that scattering degrades $V_{\mathrm{oc}}$ in $\mathrm{B}-\mathrm{C}$ $(-90 \mathrm{mV})$ while this degradation is not significant in B-B. This result suggests that scattering with phonon increases the broadening factor. However, this point deserves further investigations, which cannot be conducted in the present work due to huge numerical burden.

\subsection{Optimization of the tunnel barrier}

The broadening factor may suggest that in the B-B system better characteristics are expected if we assume a thick-tunnel barrier between the QW and the contact. Indeed, due to strong tunnel reflection, life-time increases and then broadening of the excited state decreases. Figure 6 shows the evolution of both $I_{\mathrm{sc}}$ and $V_{\mathrm{oc}}$ versus the tunnel barrier thickness $t_{B}$ in the $\mathrm{B}-\mathrm{B}$ system (remember that in Fig. $\left.2 \mathrm{a}, t_{B}=2 \mathrm{~nm}\right)$. For $t_{B}$ lower than $2 \mathrm{~nm}$, the reduction of $V_{\mathrm{oc}}$ is due to the broadening factor. At the same time the larger current with the ultra-thin barrier suggests another behavior like a reduction of the tunnel reflection improving the excitation-collection trade-off. This is confirmed for the thick barriers where $I_{\mathrm{sc}}$ and then the collection are strongly degraded by such a reflection. With a barrier thicker than $3 \mathrm{~nm}$ the excitation-collection trade-off is strongly degraded. In the same time, $V_{\text {ос }}$ no more increases for barrier thicker than $2 \mathrm{~nm}$ since the excited state is thin enough $(\leq 5 \mathrm{meV})$ to cancel the broadening factor. For an optimal tunnel barrier both broadening factor and excitation-collection trade-off should be considered. With the parameters considered in this work the trade-off is optimal for a $2 \mathrm{~nm}$-thick tunnel barrier. In case of a barrier made of an other material with an effective mass $m^{*}$ (in free electron mass) and a rectangular tunnel barrier offset $\Delta$ (in eV), this tunneling coupling and then the corresponding excitation-collection trade-off should be conserved if the thickness of the barrier (in $\mathrm{nm}$ ) is given by $t_{b}=\frac{0.43}{\sqrt{m * \Delta}}$. 


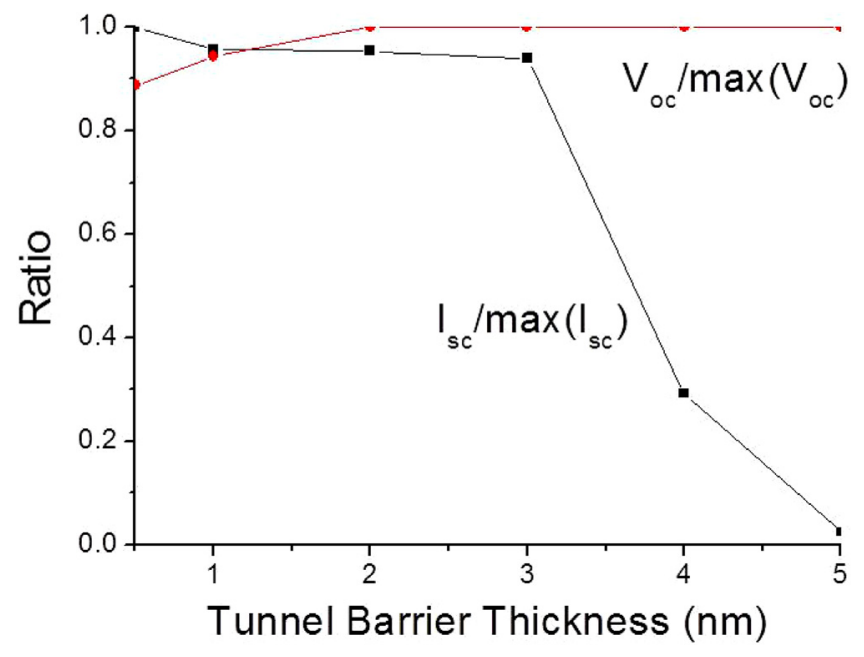

Fig. 6. $I_{\mathrm{sc}} / \max \left(I_{\mathrm{sc}}\right)$ and $V_{\mathrm{oc}} / \max \left(V_{\mathrm{oc}}\right)$ versus the thickness of the tunnel barrier $t_{B}$. Assuming a thick barrier excited states are very thin and energy mesh has to be also very thin. From a computational point of view we cannot conduct the calculation for tunnel thicker than $3 \mathrm{~nm}$ assuming the transverse dispersion. This calculation has then been conducted only for a transverse wave vector equals to zero.

\section{Conclusion}

We have shown that a broadening factor degrades the voltage in intraband system by improving the photon emission. To avoid this degradation a bound-to-bound system in which the electrons are collected by tunneling is well adapted. Moreover, such a thin tunnel barrier improves the excitation-collection trade-off due to a higher wave-function overlap. On the other hand, a too thick tunnel barrier degrades this excitation-collection trade-off due to strong tunnel reflection. It is then necessary to choose a tunnel barrier thick enough to cancel the broadening factor and to improve the wave function overlap, but thin enough to preserve the excitationcollection trade-off. We finally propose an equation, which allows calculating the thickness of the optimal barrier versus the effective mass and the tunnel barrier offset.

Nicolas Cavassilas and Daniel Suchet thank the Japan Society for the Promotion of Science (JSPS) for financial support.

\section{Author contribution statement}

N.C. used the homemade quantum transport model developed jointly by N.C., M.B. and F.B. D.S. with N.C. developed the analytical model for the broadening factor. The idea of this study come from discussions at Tokyo University with A.D., J.F.G., M.S. and Y.O. All authors have given approval to the final version of the manuscript.

\section{References}

1. A. Luque, A. Mart, C. Stanley, Nat. Photon. 6, 146 (2012)

2. A. Luque, A. Mart, Phys. Rev. Lett. 78, 5014 (1997)

3. W. Shockley, H.J. Queisser, J. Appl. Phys. 32, 510 (1961)

4. T. Sogabe, Y. Shoji, M. Ohba, K. Yoshida, R. Tamaki, H.-F. Hong, C.-H. Wu, C.-T. Kuo, S. Tomi, Y. Okada, Sci. Rep. 4 srep04792 (2014)

5. Y. Okada, N.J. Ekins-Daukes, T. Kita, R. Tamaki, M. Yoshida, A. Pusch, O. Hess, C.C. Phillips, D.J. Farrell, K. Yoshida, et al., Appl. Phys. Rev. 2, 021302 (2015)

6. A. Pusch, M. Yoshida, N.P. Hylton, A. Mellor, C.C. Phillips, O. Hess, N.J. Ekins-Daukes, Progr. Photovoltaics 24, 656 (2016)

7. N. López, K.M. Yu, T. Tanaka, W. Walukiewicz, Adv. Energy Mater. 6, 1501820 (2016)

8. B.F. Levine, A. Zussman, S.D. Gunapala, M.T. Asom, J.M. Kuo, W.S. Hobson, J. Appl. Phys. 72, 4429 (1992)

9. G. Bastard, Wave mechanics applied to semiconductor heterostructures, in Monographies de Physique (Les Editions de Physique, 1988)

10. N. Cavassilas, F. Michelini, M. Bescond, J. Renew. Sustain. Energy 6, 011203 (2013)

11. N. Cavassilas, F. Michelini, M. Bescond, J. Comput. Electron. 15, 1233 (2016)

12. A. Delamarre, D. Suchet, M. Sugiyama, N. Cavassilas, Y. Okada, J.-F. Guillemoles, Proc. SPIE 10527, 105270R (2018)

13. M. Yoshida, N.J. Ekins-Daukes, D.J. Farrell, C.C. Phillips, Appl. Phys. Lett. 100, 263902 (2012)

14. S.-W. Kim, J.P. Zimmer, S. Ohnishi, J.B. Tracy, J.V. Frangioni, M.G. Bawendi, J. Am. Chem. Soc. 127, 10526 (2005)

15. D. Sato, J. Ota, K. Nishikawa, Y. Takeda, N. Miyashita, Y. Okada, J. Appl. Phys. 112, 094305 (2012)

16. S. Tomi, T. Sogabe, Y. Okada, Progr. Photovoltaics 23, 546 (2015)

17. U. Aeberhard, U. Rau, Phys. Rev. Lett. 118, 247702 (2017)

18. T. Markvart, Phys. Status Solidi a 205, 2752 (2008)

19. L.C. Hirst, N.J. Ekins-Daukes, Progr. Photovoltaics 19, 286 (2011)

20. J. Yao, T. Kirchartz, M.S. Vezie, M.A. Faist, W. Gong, Z. He, H. Wu, J. Troughton, T. Watson, D. Bryant, et al., Phys. Rev. Appl. 4, 014020 (2015)

21. U. Rau, B. Blank, T.C. Müller, T. Kirchartz, Phys. Rev. Appl. 7, 044016 (2017)

Cite this article as: Nicolas Cavassilas, Daniel Suchet, Amaury Delamarre, Fabienne Michelini, Marc Bescond, Yoshitaka Okada, Masakazu Sugiyama, Jean-Francois Guillemoles, Beneficial impact of a thin tunnel barrier in quantum well intermediate-band solar cell, EPJ Photovoltaics 9, 11 (2018) 